\title{
THE AUTO IGUSA-ZETA FUNCTION OF A PLANE CURVE SINGULARITY IS RATIONAL.
}

\author{
ANDREW R. STOUT
}

\section{INTRODUCTION}

The auto Igusa-zeta function

$$
\zeta_{X, p}(t) \in \mathbf{G r}\left(\operatorname{Var}_{\kappa(p)}\right)\left[\mathbb{L}^{-1}\right][[t]]
$$

was introduced in Sch2]. In this paper, Schoutens showed that if $X=\mathbb{A}_{k}^{d}$ where $k$ is an algebraically closed field and $p \in X$ is a closed point, then

$$
\zeta_{X, p}(t)=\mathbb{L}^{-\operatorname{dim}(X)}(1-t)^{-1} .
$$

In $\underline{\mathrm{St}}$, I easily generalized this to obtain the following:

Proposition 1.1. Let $k$ be any field and let $p$ be any point of $X$. Let $f: X \rightarrow Y$ be a morphism of $k$-schemes which is étale at $p \in X$, then

$$
\zeta_{X, p}(t)=\zeta_{Y, f(p)}(t)
$$

In particular, if $X$ is smooth at $p$, then

$$
\zeta_{X, p}(t)=\mathbb{L}^{-\operatorname{dim}_{p}(X)}(1-t)^{-1} \in \mathbf{G r}\left(\operatorname{Var}_{\kappa(p)}\right)\left[\mathbb{L}^{-1}\right][[t]],
$$

where $\operatorname{dim}_{p}(X):=\operatorname{krull}-\operatorname{dim}\left(\mathcal{O}_{X, p}\right)$. It is theorized further in loc. cit. that the auto Igusa-zeta function $\zeta_{X, p}(t)$ is a perfect local invariant - i.e., we propose the following conjecture

ConjeCture 1.2. Let $k$ be any field and let $X$ and $Y$ be any separated $k$-schemes of finite type. If there are points $p \in X$ and $q \in Y$ such that $\zeta_{X, p}(t)=\zeta_{Y, q}(t)$, then $(X, p)$ is analytically isomorphic to $(Y, q)$.

In particular, a weak form of this conjecture1 will be that if

$$
\zeta_{X, p}(t)=\mathbb{L}^{-\operatorname{dim}_{p}(X)}(1-t)^{-1},
$$

then $X$ is smooth at $p$. We show in 3.4 that this conjecture is true when $X$ is a plane curve.

Subject Classification: $14 \mathrm{H} 20$ \& 14E18. The author was partially supported by a PSC-CUNY Grant, (Tradional A) cycle 46.

${ }^{1}$ One also expect that if $[X] \zeta_{X, p}(t)=\Theta_{X, l}(t)$, then $X$ is smooth at $p$. Here, $\theta_{X, l}(t)$ is the classical motivic Igusa-zeta function. 
In $[\mathrm{St}$, I explicitly computed the auto Igusa-zeta function in the following three cases: 1) $X$ is the node $x y=0,2) X$ is the nodal cubic $y^{2}=x^{3}-x^{2}$, and 3) $X$ is the cuspidal cubic $y^{2}=x^{3}$. Furthermore, I indicated how to do such computations for more complicated singular points of algebraic curves. In summary, there is computational evidence that:

- Conjecture 1.2 will be true.

- $[X] \zeta_{X, p}(t)=\Theta_{X, l}(t)$ iff $X$ is a smooth.

- $\zeta_{X, p}(t)$ is a rational function.

- Every pole of $\zeta_{X, p}(t)$ is also a pole of $\Theta_{X, \mathrm{r}}(t)$.

Indeed, we will show in this paper that each of these bullets will hold when $X$ is a curve on a smooth surface over an algebraically closed field of characteristic zero.

\section{Auto-ARC spaces as fibers of Generalized ARC SPACES}

Let $k$ be any field and let $C$ be a variety2. Let $p \in C$ with residue field $\kappa(p)$ and consider the $n$-th jet $J_{p}^{n} C:=\operatorname{Spec}\left(\mathcal{O}_{C, p} / \mathcal{M}_{p}^{n}\right)$. For a separated $\kappa(p)$-scheme of finite type, we define $\nabla_{J_{p}^{n} C} X$ to be the scheme which represents the functor Aff $_{\kappa(p)} \rightarrow$ \$ets defined by

$$
\operatorname{Spec}(A) \mapsto \operatorname{Mor}_{\mathbb{S} \subset \mathfrak{h}_{\kappa(p)}}\left(J_{p}^{n} C \times_{\kappa(p)} \operatorname{Spec}(A), X\right)
$$

The canonical surjection of rings $\mathcal{O}_{C, p} / \mathcal{M}_{p}^{n} \rightarrow \kappa(p)$, induces a morphism $\pi_{n}$ : $\nabla_{J_{p}^{n} C} X \rightarrow X$. When $X=J_{p}^{n} C$, we reserve the notion $\mathcal{A}_{n}(C, p)$ for $\nabla_{J_{p}^{n} C} J_{p}^{n} C$ and call it the auto arc space of $C$ at the point $p$ at level $n$. It is a basic example of an auto arc space which were introduced and studied $\S 3, \S 8$ and Appendix A of [St].

LEMma 2.1. Let $C$ be any variety, $p$ a point of $C$, and $\kappa(p)$ the residue field at $p$. Let $U$ be the smooth locus of a variety $X$ over $\kappa(p)$, then $\pi_{n}: \nabla_{J_{p}^{n} C} X \rightarrow X$ is a piecewise trivial fibration over $U$.

Proof. By Theorem 4.4 of [Sch2], $\pi_{n}^{-1}(U) \cong \nabla_{J_{p}^{n} C} U$. Using étale charts of $U$, it is straightforward to show $\nabla_{J_{p}^{n} C} U \cong U \times \mathbb{A}_{\kappa(p)}^{m}$ where $m=\operatorname{dim}_{p}(C)\left(\ell\left(J_{p}^{n} C\right)-1\right)$.

Lemma 2.2. Let $C$ be a be a variety and let $p$ be a point of $C$. Then,

$$
\pi_{n}^{-1}(p)^{\text {red }} \cong \mathcal{A}_{n}(C, p)^{\text {red }}
$$

where $\pi_{n}: \nabla_{J_{p}^{n} C} C \rightarrow C$.

Proof. This follows by applying reduction to the isomorphism given by Lemma 4.9 of Sch2].

\footnotetext{
${ }^{2}$ We take this to mean that $C$ is a separated scheme of finite type over $k$ which is also reduced
} 
THE AUTO IGUSA-ZETA FUNCTION OF A PLANE CURVE SINGULARITY IS RATIONAL. 3

LEMma 2.3. Let $C$ be a variety with only one singular point $p \in C$. Then,

$$
\left[\nabla_{J_{p}^{n} C} C\right]=[C \backslash\{p\}] \mathbb{L}^{m}+\left[\mathcal{A}_{n}(C, p)\right]
$$

in $\mathbf{G r}\left(\operatorname{Var}_{k(p)}\right)$ where $m=\operatorname{dim}_{p}(X)\left(\ell\left(J_{p}^{n} C\right)-1\right)$.

Proof. This is a direct concequence of Lemma 2.1 and Lemma 2.2 .

The generalized motivic zeta function $\Theta_{C, \mathfrak{x}}(t)$ and the auto Igusa-zeta function 3 $\zeta_{C, p}(t)$ were defined in $\S 7$ and $\S 3$ of [St], respectively.

THEOREM 2.4. Let $J_{p}^{\infty} C$ denote the formal scheme defined by $\lim _{n} J_{p}^{n} C$ where $C$ is a variety with only one singular point $p$. In this case, the motivic zeta function of $C$ with respect to $J_{p}^{\infty} C$ decomposes as

$$
\Theta_{C, J_{p}^{\infty} C}(t)=\mathbb{L}^{-\operatorname{dim}_{p}(C)} \frac{[C]-1}{1-t}+\zeta_{C, p}(t)
$$

in $\operatorname{Gr}\left(\operatorname{Var}_{\kappa(p)}\right)\left[\mathbb{L}^{-1}\right][[t]]$.

Proof. This follows from the definition of the motivic zeta function (Definition 7.1 of $[\mathrm{St}$ ] ) and Lemma 2.3 .

ExAmple 2.5. Let $C$ be the cuspidal cubic defined by $y^{2}=x^{3}$. This has one singularity at the origin $O=(0,0)$. We immediately arrive at

$$
\Theta_{C, J_{O}^{\infty} C}(t)=\mathbb{L}^{-1} \frac{\left[\mathbb{G}_{m}\right]}{1-t}+\zeta_{C, O}(t) .
$$

I showed in $\S 7$ of $\mathrm{St}$ that $\zeta_{C, O}(t)$ is rational - in fact, we arrived at the following explicit formula:

$$
\zeta_{C, O}(t)=\mathbb{L}^{-1}+\mathbb{L} t+\mathbb{L}^{2} t^{2}+\frac{\left(\mathbb{L}^{7}-\mathbb{L}^{6}\right) t^{3}+\mathbb{L}^{7} t^{4}+\mathbb{L}^{7} t^{7}}{\left(1-\mathbb{L} t^{3}\right)(1-t)}
$$

REMARK 2.6. We expect that $\Theta_{C, \mathfrak{x}}(t)$ is rational for any scheme $C$ and any filtered colimit of fat points $x$. In particular, we expect that the auto Igusa-zeta function $\zeta_{C, p}(t)$ is rational and that every pole of of $\zeta_{C, p}(t)$ is also a pole of the classical motivic zeta function $\Theta_{C, \mathfrak{l}}(t)$ where $\mathfrak{l}=\lim _{n} \operatorname{Spec}\left(\kappa(p)[t] /\left(t^{n}\right)\right)$.

\section{Rationality of the auto Igusa-Zeta function of a Plane Curve.}

In $\underline{\mathrm{St}}$, we used a computational approach to find explicit formulas for the auto Igusa-zeta function of a few algebraic curves and arrived at a basic idea for how the auto Igusa-zeta function behaves for curves. Namely, it appears that finding the expansion of $\zeta_{C, p}(t)$ in terms of the classical motivic zeta function is closely related to its normalization. In this section, we will gain a better picture of why exactly that must be the case.

\footnotetext{
${ }^{3}$ There it was denoted as $\bar{\zeta}_{C, p}(t)$ where the bar was written to clarify that it came from applying reduction to a more general type of zeta function.
} 
Assumption 3.1. In this section, we work relative to the following set-up. We let $C$ be a curve on a smooth surface over an algebraically closed field $k$. We let $\gamma: \bar{C} \rightarrow C$ be the normalization of $C$. We fix a point $p$ of $C$, and we assume that the characteristic of $k$ does not divide $\operatorname{mult}_{q}(\bar{C})$ for all $q \in \gamma^{-1}(p)$. Note that by a curve, we mean that $C$ is a reduced separated $k$-scheme of finite type which has pure dimension 1. Also, note that the last condition means that $\operatorname{char}(k)$ will not divide $\operatorname{mult}_{p}(C)$.

REMARK 3.2. If we start with a smooth point $p$ of $C$, then $\gamma: \bar{C} \rightarrow C$ will be an isomorphism away from the exceptional locus $\gamma^{-1}\left(C_{\text {sing }}\right)$. In particular, $\hat{\mathcal{O}}_{C, p} \cong \hat{\mathcal{O}}_{\bar{C}, q} \cong k[[t]]$. This immediately implies that $J_{p}^{\infty} C \cong \operatorname{Spf}(k[[t]])$ and for any $k$-scheme $X, \nabla_{\mathfrak{l}} X=\lim _{\mathfrak{L}_{n}} \nabla_{J_{p}^{n} C} X$ is the usual arc space. Note that $\nabla_{\mathfrak{l}} X$ is most commonly denoted by $\mathcal{L}(X)$ in the literature (cf. [DL1]). In this paper, we are mostly concerned with the case when $p$ is a singular point of $C$.

3.1. Preliminaries on the normalization of a curve. Now, consider its normalization $\gamma: \bar{C} \rightarrow C$. Let $p$ be a point of $C$. For each branch $q \in \gamma^{-1}(p)$, the Newton-Puiseux Theorem states that given local coordinates $(x, y)$ of the point $q$, we have

$$
\begin{aligned}
& x=t^{r} \\
& y=\sum_{i=1}^{\infty} a_{i} t^{i}
\end{aligned}
$$

with $a_{i} \in k$. In fact, if we let $f(x, y) \in k[x, y]$ be such that $f(x, y)=0$ defines $C$, then the image $F(x, y)$ of $f(x, y)$ in $k[[x, y]]$ factors as $F(x, y)=u(x, y) \prod_{j=1}^{s} G_{j}(x, y)$ where $u(x, y)$ is a unit and the $G_{j}(x, y)$ are distinct irreducible power series. The power series $G_{j}(x, y)$ are sometimes also referred to as the branches of $C$. In fact, using the Newton-Puiseux Theorem 4 there is a ring homomorphism $\varphi_{j}: k[[x, y]] \rightarrow$ $k[[t]]$ such that

$$
\varphi_{j}\left(G_{j}(x, y)\right)=G_{j}\left(t^{r_{j}}, \sum_{i=1}^{\infty} a_{i, j} t^{i}\right)=0
$$

which therefore induces an embedding $k[[x, y]] /\left(G_{j}(x, y)\right) \hookrightarrow k[[t]]$. In other words, if we let $\hat{\mathcal{O}}_{\bar{C}, q}$ be the completion of the local ring $\mathcal{O}_{\bar{C}, q}$ with $q \in \gamma^{-1}(p)$ and let $\hat{\mathcal{O}}_{C, p}$ be the completion of the local ring $\mathcal{O}_{C, p}$, which is isomorphic to $k[[x, y]] /(F(x, y))$, then it follows that there is an embedding of rings

$$
\hat{\mathcal{O}}_{C, p} \hookrightarrow \hat{\mathcal{O}}_{\bar{C}, q}
$$

making $\hat{\mathcal{O}}_{\bar{C}, q}$ a finite $\hat{\mathcal{O}}_{C, p}$-algebra.

In particular, if $C$ is unibranched (meaning that $s=1$ ), then $\hat{\mathcal{O}}_{C, p}$ embeds into $k[[t]]$ as a subring and this ring embedding is induced by $\varphi_{1}$. Moreover, if $s \geq 1$, we

\footnotetext{
${ }^{4}$ This is where our assumptions that $k$ is algebraically closed and that char $(k)$ does not divide any $r_{j}$ are being used.
} 
THE AUTO IGUSA-ZETA FUNCTION OF A PLANE CURVE SINGULARITY IS RATIONAL. 5

have an embedding $\varphi: \hat{\mathcal{O}}_{C, p} \hookrightarrow \prod_{j=1}^{s} k[[t]]$ where $\varphi=\prod_{j=1}^{s} \varphi_{j}$. On the geometric side, $\gamma_{n}: E_{n} \rightarrow \operatorname{Spec}\left(\mathcal{O}_{C, p} / \mathcal{M}_{p}^{n}\right)$ where $E_{n}=\bar{C} \times{ }_{k} \operatorname{Spec}\left(\mathcal{O}_{C, p} / \mathcal{M}_{p}^{n}\right)$. Taking filtered colimits in formal schemes, which is the same as completing the integral closure of $\mathcal{O}_{C, p}$ with respect to the Jacobson radical, gives a formal scheme

$$
\hat{E} \cong \sqcup_{j=1}^{s} \operatorname{Spf}(k[[t]])
$$

and a surjective morphism $\hat{\gamma}: \hat{E} \rightarrow \operatorname{Spf}\left(\hat{\mathcal{O}}_{C, p}\right)$ such that $\hat{\gamma}=\operatorname{Spf}(\varphi)$. The morphism $\hat{\gamma}$ will be called the uniformization morphism of the analytic germ $(C, p)$.

Example 3.3. Let $r>1$ and $n>r$ with $n$ and $r$ coprime. Consider the curve defined by $f(x, y)=y^{r}-x^{n}=0$, which has a singular point at the origin $O$. This is a unibranched curve, and the homomorphism $\varphi_{1}: k[[x, y]] \rightarrow k[[t]]$ defined by $\varphi_{1}(x)=t^{r}$ and $\varphi_{1}(y)=t^{n}$ induces an embedding $\hat{\mathcal{O}}_{C, O} \hookrightarrow k[[t]]$. Moreover, one can show that $\gamma: \bar{C} \rightarrow C$ is bijective as it is given by $t \mapsto\left(t^{r}, t^{n}\right)$.

3.2. Case of a unibranched curve. In this subsection, we focus entirely on the unibranched case as outlined above - i.e., we let $C$ be a plane curve with only one singular point, which we may assume without loss of generality occurs at the origin $O$, such that the normalization $\gamma: \bar{C} \rightarrow C$ is bijective. Then, as outlined above, $\hat{\mathcal{O}}_{C, O} \cong k[[x, y]] / F(x, y)$ embeds into $\hat{\mathcal{O}}_{\bar{C}, \gamma^{-1}(O)} \cong k[[t]]$ via the morphism $\varphi: k[[x, y]] \rightarrow k[[t]]$ defined $\varphi(x)=t^{r}$ and $\varphi(y)=\sum_{i=1}^{\infty} a_{i} t^{i}$.

By 1.89 and Theorem 1.96 of [JK], we have that $r=$ mult $_{O} C$. Thus, without loss of generality, we may assume that $y^{r}$ occurs as a term in $F(x, y)$ with constant nonzero coefficient. In other words, if we let $F_{r}$ be the homogeneous term of $F(x, y)$ of degree $r$, then $F_{r}$ is also the homogeneous term of $F(x, y)$ of minimal degree.

The ring homomorphism $\varphi: k[[x, y]] \rightarrow k[[t]]$ will induce a homomorphism $\varphi_{n}$ : $k[[x, y]] /(x, y)^{n} \hookrightarrow k[[t]] /\left(t^{r n}\right)$. Note the only thing to check here is that $y^{n} \mapsto 0$ under $\varphi_{n}$ but this is indeed the case as the first nonvanishing term of $\sum_{i=1}^{\infty} a_{i} t^{i}$ is $a_{m} t^{m}$ with $m \geq n$. This follows from the set-up in the preceding paragraph and Corollary 2.2 .8 by [EA]. We now have enough to prove the following theorem:

TheOrem 3.4. Let $X$ be a $k$-variety and let $(C, p)$ be a pointed unibranched curve satisfying Assumption 3.1. Let $r=$ mult $_{p}(C)$. Then

(1) The generalized arc space $\nabla_{J_{p}^{n} C} X$ is a closed subvariety of $\nabla_{\mathfrak{l}_{r n}} X$.

(2) The natural truncation morphism $\pi_{n}: \nabla_{J_{p}^{n} C} X \rightarrow \nabla_{J_{p}^{n-1} C} X$ is induced by restricting the natural truncation morphism $\nabla_{\mathfrak{l}_{r n}} X \rightarrow \nabla_{\mathfrak{l}_{r(n-1)}} X$.

(3) The projective limit $\nabla_{J_{p}^{\infty} C} X:=\lim _{n_{n}} \nabla_{J_{p}^{n} C} X$ is a closed cylinder of $\nabla_{\mathfrak{l}} X$.

(4) There is an associated zeta function $Z_{r}(t)$ to $\nabla_{J_{p}^{\infty} C} X$.

(5) If $X$ has a resolution of singularities (e.g., $\operatorname{char}(k)=0)$, then $Z_{r}(t)$ is rational - i.e., $Z_{r}(t) \in \mathbf{G r}\left(\operatorname{Var}_{k}\right)\left[\mathbb{L}^{-1}\right](t)$.

Proof. We have shown that there is a ring embedding $\mathcal{O}_{C, p} / \mathcal{M}_{p}^{n} \hookrightarrow k[t] /\left(t^{r n}\right)$ for all $n>0$. This immediately implies (1) and (2). To prove (3), it is enough to 
work with $k$ points. We see that the image of the ring embedding $\varphi: \hat{\mathcal{O}}_{C, p} \hookrightarrow k[[t]]$ can be described set-theoretically as the collection of arcs $a_{0}+\sum_{i \geq r} a_{i} t^{i}$ generated as a $k$-algebra by $1, \varphi(x)$, and $\varphi(y)$, with $\operatorname{ord}_{t}(\varphi(x))=r$ and $\operatorname{ord}_{t}(\varphi(y))=m$. Let $c$ be the conductor-i.e., the minimal natural number so that $\left(t^{c}\right) k[[t]] \subset \hat{\mathcal{O}}_{C, p}$. It follows that for any $n \in \mathbb{N}$ such that $n r \geq c$,

$$
\nabla_{J_{p}^{\infty} C} X=\pi_{n r}^{-1}\left(\nabla_{J_{p}^{n} C} X\right) .
$$

For (4), consider the generating function

$$
\mathbb{L}^{-1} P\left(\mathbb{L}^{-1} t\right)=\sum_{n=0}^{\infty}\left[\pi_{n+1}\left(\nabla_{J_{p}^{\infty} C} X\right)\right] \mathbb{L}^{-(n+1)} t^{n},
$$

where $P(s)$ is the motivic Poincaré series of $\nabla_{J_{p}^{\infty} C} X$. This is a rational function by Theorem 5.4 of DL2]. Thus, the sum of the $r$ th terms of $\mathbb{L}^{-1} P\left(\mathbb{L}^{-1} t\right)$, which we denote here by $Z_{r}(t)$, is also rational.

Corollary 3.5. Let $C$ be a unibranched curve satisfying Assumption 3.1. Let $r=\operatorname{mult}_{p}(C)$. Then,

(1) The auto-arc space $\mathcal{A}_{n}(X, p)^{\text {red }}$ is a closed subvariety of $\nabla_{\mathfrak{l}_{r n}} C$.

(2) The natural truncation morphism $\pi_{n}: \mathcal{A}_{n}(C, p)^{\mathrm{red}} \rightarrow \mathcal{A}_{n-1}(C, p)^{\mathrm{red}}$ is induced by restricting the natural truncation morphism $\nabla_{\mathfrak{l}_{r n}} C \rightarrow \nabla_{\mathfrak{l}_{r(n-1)}} C$.

(3) The infinite auto-arc space $\mathcal{A}(C, p):=\lim _{n}\left(\mathcal{A}_{n}(C, p)\right)^{\mathrm{red}}$ is a closed cylinder of $\nabla_{\mathfrak{l}} C$.

(4) There is an associated zeta function $Z_{r}^{a}(t)$ to $\mathcal{A}(C, p)$ which is rational i.e., $Z_{r}^{a}(t) \in \mathbf{G r}\left(\operatorname{Var}_{k}\right)\left[\mathbb{L}^{-1}\right](t)$.

Proof. Parts (1), (2), and (3) immediately follow from Lemma 2.3 and Theorem 3.4. Part (4) is also the same except that we let $Z_{r}^{a}(t)$ denote the $r$ th terms of $\mathbb{L}^{-1} P\left(\mathbb{L}^{-1} t\right)$ where $P(s)$ is now the motivic Poincaré series of $\mathcal{A}(C, p)$.

Let $C$ be any curve and let $p$ be a point of $C$. The Hilbert-Samuel function $\ell\left(\mathcal{O}_{C, p} / \mathcal{M}_{p}^{n}\right)$ is given by a linear polynomial $P(n)=e_{0}(C, p) n+e_{1}(C, p)$ for sufficiently large $n$, where $e_{i}(C, p) \in \mathbb{Z}$. The polynomial $P(n)$ is sometimes called the Hilbert-Samuel polynomial of $C$ at $p$, and the number $e_{0}(C, p)$ is called the HilbertSamuel multiplicity of $C$ at $p$. It is well-known (e.g., see the beginning of Chapter 4 , Section 3 of $[\mathrm{WF}])$ that $e_{0}(C, p)$ corresponds with the usual geometric notion of multiplicity used above -i.e., we have that $e_{0}(C, p)=r$ where $r$ is the number used in the work above. This immediately implies the following:

COROLlary 3.6. Let $k$ be an algebraically closed field and let $X$ be a k-variety admitting a resolution of singularities. Let $(C, p)$ be a pointed unibranched curve satisfying Assumption [3.1. Then, $\Theta_{X, J_{p}^{\infty} C}\left(t^{r}\right)-\mathbb{L}^{-e_{1}(C, p)} Z_{r}(t)$ and $\zeta_{C, p}\left(t^{r}\right)-$ $\mathbb{L}^{-e_{1}(C, p)} Z_{r}^{a}(t)$ are polynomials (i.e., elements of $\mathbf{G r}\left(\operatorname{Var}_{k}\right)[t]$ ) of degree at most 
THE AUTO IGUSA-ZETA FUNCTION OF A PLANE CURVE SINGULARITY IS RATIONAL. 7

$r c$, where $c$ is the conductor of $C$. In particular, $\Theta_{X, J_{p}^{\infty} C}(t)$ and $\zeta_{C, p}(t)$ are both rational functions.

3.3. The multibranched case. Let $X$ be any scheme. We will use the short hand $X^{s}$ for the fiber product $X \times_{k} \cdots \times_{k} X$ (s times). Using the uniformization morphism and some facts about arc spaces, we arrive at the following analogue of Theorem 3.4 for the multibranched case.

THEOREM 3.7. Let $X$ be a k-variety and let $(C, p)$ be a pointed curve (not necessarily unibranched) satisfying Assumption 3.1. Let $r_{i}=$ mult $_{q_{i}}(\bar{C})$ where $q_{i}$ are the branches of $C$ at $p$. Then,

(1) The generalized arc space $\nabla_{J_{p}^{n} C} X$ is a closed subvariety of $\prod_{i=1}^{s} \nabla_{\mathfrak{l}_{r_{i} n}} X$.

(2) The natural truncation morphism $\pi_{n}: \nabla_{J_{p}^{n} C} X \rightarrow \nabla_{J_{p}^{n-1} C} X$ is induced by restricting the natural truncation morphism $\prod_{i=1}^{s} \nabla_{\mathfrak{l}_{r_{i} n}} X \rightarrow \prod_{i=1}^{s} \nabla_{\mathfrak{l}_{r_{i}(n-1)}} X$.

(3) The projective limit $\nabla_{J_{p}^{\infty} C} X:=\lim _{n} \nabla_{J_{p}^{n} C} X$ is a closed cylinder of $\nabla_{\mathfrak{l}} X^{s}$.

(4) There is an associated zeta function $Z_{r}(t)$ to $\nabla_{J_{p}^{\infty} C} X$.

(5) If $X$ admits a resolution of singularities, then $Z_{r}(t)$ is rational $-i . e ., Z_{r}(t) \in$ $\operatorname{Gr}\left(\operatorname{Var}_{k}\right)\left[\mathbb{L}^{-1}\right](t)$.

Proof. Let $C$ be a curve on a smooth surface and let $p$ be a point on $C$. Let $s$ be the cardinality of $\gamma^{-1}(p)$ where $\gamma: \bar{C} \rightarrow C$ is the normalization. Consider the uniformization homomorphism $\hat{\mathcal{O}}_{C, p} \hookrightarrow \sqcup_{i=1}^{s} k[[t]]$ defined by $\sqcup_{i=1}^{s} \varphi_{i}$ with $\varphi_{i}(x)=$ $t^{r_{i}}$ and $\varphi_{i}(y)=\sum_{j=1} a_{j, i} t^{j}$. This induces the closed embedding of (1) and (2) quickly follows as well.

For (3), note that

$$
\nabla_{\mathfrak{l}} X^{s}=\nabla_{\mathfrak{l}}\left(\prod_{i=1}^{s} X\right) \cong \prod_{i=1}^{s} \nabla_{\mathfrak{l}} X
$$

Part (3) follows immediately from the fact that $\lim _{n} \nabla_{\mathfrak{l}_{r_{i}}} X \cong \nabla_{\mathfrak{l}} X$, the proof of Theorem 3.4. and the fact that a finite fiber product of closed cylinders is a closed cylinder. The proof for part (4) follows from (3) in exactly the same way as in the proof of Theorem 3.4 .

For part (4) and (5), consider the generating function

$$
\mathbb{L}^{-1} P\left(\mathbb{L}^{-1} t\right)=\sum_{n=0}^{\infty}\left[\pi_{n+1}\left(\nabla_{J_{p}^{\infty} C} X\right)\right] \mathbb{L}^{-(n+1)} t^{n}
$$

where $P(s)$ is the motivic Poincaré series of $\nabla_{J_{p}^{\infty} C} X$. This is a rational function by Theorem 5.4 of DL2 provided $X$ has a resolution of singularities. Thus, the sum of its $r$ th terms, which we denote by $Z_{r}(t)$, is rational. More explicitly, we choose $r=\operatorname{mult}_{p}(C)=r_{1}+\cdots+r_{s}$. We have

$$
Z_{r}(t)=\sum_{n=m}^{\infty}\left[\nabla_{J_{p}^{(n+1) r} C} X\right] \mathbb{L}^{-(n+1) r} t^{n r}+\text { lower order terms in } t
$$

for sufficiently large $m$. 
REMARK 3.8. Although we do not make use of it, one may also consider the generating function

$$
G(t)=\mathbb{L}^{-s} P_{1}\left(\mathbb{L}^{-1} t\right) P_{2}\left(\mathbb{L}^{-1} t\right) \cdots P_{s}\left(\mathbb{L}^{-1} t\right)
$$

where $P_{i}(w)$ is the motivic Poincaré series of $C_{i}$ where $C_{i}$ is the image of $\nabla_{J_{p}^{\infty} C} X$ under the natural projection onto the $i$ th factor of $\prod_{j}^{s} \nabla_{\mathfrak{l}} X$. This will also be rational provided $X$ has a resolution of singularities.

Corollary 3.9. Let $(C, p)$ be a pointed curve (not necessarily unibranched) satisfying Assumption 3.1. Let $r_{i}=$ mult $_{q_{i}}(\bar{C})$ where $q_{i}$ are the branches of $C$ at $p$. Then,

(1) The auto-arc space $\mathcal{A}_{n}(X, p)^{\text {red }}$ is a closed subvariety of $\prod_{i=1}^{s} \nabla_{\mathfrak{l}_{r_{i} n}} X$.

(2) The natural truncation morphism $\pi_{n}: \mathcal{A}_{n}(C, p)^{\text {red }} \rightarrow \mathcal{A}_{n-1}(C, p)^{\text {red }}$ is induced by restricting the natural truncation morphism $\prod_{i=1}^{s} \nabla_{\mathfrak{l}_{r_{i} n}} X \rightarrow$ $\prod_{i=1}^{s} \nabla_{\mathfrak{l}_{r_{i}(n-1)}} X$.

(3) The infinite auto-arc space $\mathcal{A}(C, p):=\lim _{n}\left(\mathcal{A}_{n}(C, p)\right)^{\mathrm{red}}$ is a closed cylinder of $\nabla_{\mathfrak{l}} C^{s}$.

(4) There is an associated zeta function $Z_{r}^{a}(t)$ to $\mathcal{A}(C, p)$ which is rational i.e., $Z_{r}^{a}(t) \in \operatorname{Gr}\left(\operatorname{Var}_{k}\right)\left[\mathbb{L}^{-1}\right](t)$.

Following the proof of Theorem 3.7 and the discussion immediately above Corollary 3.6. we quickly obtain the multibranched version of Corollary 3.6 .

COROLlary 3.10. Let $k$ be an algebraically closed field and let $X$ be a $k$-variety admitting a resolution of singularities. Let $(C, p)$ be a pointed curve satisfying Assumption 3.1] Then, $\Theta_{X, J_{p}^{\infty} C}\left(t^{r}\right)-\mathbb{L}^{-e_{1}(C, p)} Z_{r}(t)$ and $\zeta_{C, p}\left(t^{r}\right)-\mathbb{L}^{-e_{1}(C, p)} Z_{r}^{a}(t)$ are polynomials (i.e., elements of $\left.\mathbf{G r}\left(\operatorname{Var}_{k}\right)[t]\right)$ of degree at most rc, where $c$ is the conductor of $C$. In particular, $\Theta_{X, J_{p}^{\infty} C}(t)$ and $\zeta_{C, p}(t)$ are both rational functions.

3.4. Revisiting the conjecture of $\S 1$. We may now quickly make part of Conjecture 1.2 into a theorem:

THEOREM 3.11. Let $C$ be a curve on a smooth surface $S$ over an algebraically closed field $k$ of characteristic zero. Then, $C$ is smooth at $p$ if and only if

$$
\zeta_{C, p}(t)=\mathbb{L}^{-1} \frac{1}{1-t} .
$$

Proof. The forward direction is an immediate corollary to Proposition 1.1. The other direction is now (due to the rationality results in this section) also immediate. Indeed, it is impossible that

$$
\zeta_{C, p}(t)=\mathbb{L}^{-1} \frac{1}{1-t}
$$

for the mere reason that $(1-t) Z_{r}^{a}(t)$ will have a pole (in $t=\mathbb{L}^{-v}$ ) by the Motivic Monodromy Conjecture, which was proved by Loeser (cf. $[\mathrm{L}$ ) in the case of plane curves. 
THE AUTO IGUSA-ZETA FUNCTION OF A PLANE CURVE SINGULARITY IS RATIONAL. 9

\section{REFERENCES}

[EA] E. Casas-Alvero. Singularities on Plane Curves. London Mathematical Society Lecture Note Series (Book 276), Cambridge University Press, 2000.

[DL1] J. Denef \& F. Loeser. Motivic Igusa Zeta Functions. In J. Algebraic Geometry 7 no. 3 , pages 505 - 537. 1998.

[DL2] J. Denef \& F. Loeser. Germs of arcs of singular varieties and motivic integration. In Inven. Math. 135, pages 201-232, Springer, 1999.

[WF] W. Fulton. Intersection Theory. Springer, 2nd edition, 1998.

[JK] J. Kállor. Lectures on Resolution of Singularities. Annals of Mathematics Studies (Book 166), Princeton University Press, 2007.

[L] F. Loeser. Fonctions dIgusa p-adiques et polynômes de Bernstein. Amer. J. Math. 110 (1988), $1-22$.

[Sch2] H. Schoutens. Schemic Grothendieck Rings II. preprint available at websupport1.citytech.cuny.edu/faculty/hschoutens/PDF/SchemicGrothendieckRingPartII.pdf 2014.

[St] A. Stout. On the auto Igusa-zeta function of an algebraic curve. Preprint, arxiv.org (2014)

Andrew R. Stout

Dept. of Math. \& Comp. Sci.,

Bronx Community College, CP 315

2155 University Avenue

Bronx, NY 10453

E-mail address: astout@gradcenter.cuny.edu 